\title{
Adaptação de Thyrinteina arnobia em novo hospedeiro e defesa induzida por herbívoros em eucalipto
}

\author{
Anderson Mathias Holtz ${ }^{(2)}$, Hamilton Gomes de Oliveira(2) ${ }^{(2 n g e l o ~ P a l l i n i(2)}$, Jeanne Scardini Marinho(3), \\ José Cola Zanuncio(2) e Claudinei Lima Oliveira(2)
}

Resumo - O sistema de defesa induzido de plantas é ativado quando herbívoros se alimentam das mesmas. Alternativamente, herbívoros podem se adaptar a espécies de plantas filogeneticamente próximas ao seu hospedeiro de origem. O objetivo deste trabalho foi comparar o desenvolvimento de Thyrinteina arnobia em plantas de goiaba (hospedeiro de origem) e de eucalipto (hospedeiro novo) em campo. Adicionalmente, estudou-se a biologia deste inseto em laboratório fornecendo folhas de eucalipto previamente danificadas por lagartas de T. arnobia e folhas sem danos. A sobrevivência larval em eucalipto, 78,00\%, foi superior à de goiaba, 29,33\%. O ciclo larval foi de 27,90 dias em goiaba e de 30,30 dias em eucalipto. Em testes de laboratório, o ciclo larval em eucalipto limpo, 36,39 dias, foi maior do que em eucalipto danificado, 32,89 dias. A mortalidade larval em eucalipto danificado foi de $30,00 \%$ e de $10,00 \%$ em eucalipto limpo. Os resultados indicam que a goiaba não é um bom hospedeiro para T. arnobia possivelmente por apresentar um sistema mais efetivo de defesa. Embora o eucalipto possa ser um hospedeiro mais favorável ao desenvolvimento e estabelecimento do inseto, pode também reduzir populações do herbívoro ativando o seu sistema de defesa induzido.

Termos para indexação: mortalidade larval, goiaba.

\section{Adaptation of Thyrinteina arnobia to a new host and herbivore induced defense in eucalyptus}

Abstract - The induced defense system of plants is activated when herbivorous start to injury plants. Alternatively, herbivores may adapt to plants that are phylogenetically close to their original host. This work aimed at comparing the life history of Thyrinteina arnobia on guava plants (origin host) with eucalyptus (the new host) in the field. The biology of the insect in the laboratory on both eucalyptus leaves previously injured by the caterpillars of T. arnobia and on clean leaves was also studied. In the field, the larvae survivorship of T. arnobia on eucalyptus was $78.00 \%$ and on guava was $29.33 \%$. The larvae cycle was 27.90 days on guava and 30.30 days on eucalyptus. At the laboratory, tests showed that the larvae cycle on clean eucalyptus leaves lasted 36.39 days while on previously injured leaves, only 32.89 days. The larvae mortality on injured eucalyptus leaves was $30.00 \%$, and $10.00 \%$ on clean leaves. The results indicate that guava plants are not a suitable host for T. arnobia probably due to a more effective defense system. Although the eucalyptus may be a better host, it can also reduce the herbivore population by activating its herbivore induced defense system.

Index terms: larval mortality, guava.

\section{Introdução}

As interações tróficas entre planta e insetos herbívoros são importantes na determinação, distribui-

(1)Aceito para publicação em 22 de janeiro de 2003.

(2)Universidade Federal de Viçosa (UFV), Dep. de Biologia Animal, Setor de Entomologia, CEP 36571-000 Viçosa, MG E-mail: aholtz@insecta.ufv.br, hamilton@insecta.ufv.br, pallini@ufv.br, zanuncio@ufv.br, claudinei@insecta.ufv.br

(3)UFV, Dep. de Biologia Animal, Setor de Entomologia. Bolsista do CNPq. E-mail: janne@insecta.ufv.br ção e ocorrência dos organismos em ecossistema natural e manejado (Hagen et al., 1986). As plantas não são apenas passíveis aos insetos herbívoros (Agrawal, 2000); algumas de suas características, tais como compostos químicos e atributos morfológicos, influenciam na escolha alimentar do herbívoro, sua sobrevivência e fecundidade (Price, 1981).

As interações entre plantas e herbívoros são complexas e a busca do entendimento dessas interações tem sido alvo de diversos estudos (Price, 1997). 
Muitas plantas apresentam eficiente sistema de defesa induzido, ativado quando herbívoros se alimentam da planta. A ação de defesa induzida pode ocorrer por estímulos fisiológicos na planta que acentuam a produção de compostos voláteis (Vet \& Dicke, 1992) que podem servir como indicadores da presença dos herbívoros a inimigos naturais (Agrawal, 2000).

A resistência induzida da planta a ataque de insetos pragas é encontrada em muitas espécies vegetais (feijão, por exemplo) (Dicke, 1986), e é caracterizada pela redução do desempenho dos herbívoros em plantas atacadas, em relação às plantas sem nenhum tipo de dano causado por inseto (Karban, 1993).

O gênero Eucalyptus (Myrtaceae) possui cerca de 600 espécies e variedades. As primeiras plantas, oriundas da Austrália, foram trazidas para o Brasil em 1824 (Zanuncio et al., 1990). Assim, além de exótica, a eucaliptocultura é relativamente recente no Brasil em termos de adaptação de um novo gênero ou espécie em um novo ambiente.

$\mathrm{Na}$ implantação dos grandes maciços desta essência florestal, os lepidópteros desfolhadores, que antes se alimentavam exclusivamente de Mirtaceas nativas brasileiras, como a goiabeira, passaram a atacar também as plantações de eucalipto (Anjos et al., 1986). Como exemplo, Thyrinteina arnobia Stoll (Lepidoptera: Geometridae) tornou-se a mais severa praga desta essência florestal no Brasil (Anjos et al., 1986). Desta forma, acredita-se que os herbívoros que migraram das Mirtaceas brasileiras para as plantações de eucaliptos estejam em franca adaptação ao novo hospedeiro.

A adaptação dessas pragas às espécies do gênero Eucalyptus fica evidenciada porque, mesmo possuindo em sua composição grande quantidade de compostos de defesa, principalmente óleos essenciais (Fox \& Macauley, 1977), essas plantas sofrem desfolhas periódicas causadas por esses insetos (Santos et al., 2000).

O objetivo deste trabalho foi comparar o desenvolvimento de Thyrinteina arnobia em plantas de goiaba (hospedeiro de origem) e de eucalipto (hospedeiro novo), no campo, e estudar a biologia deste inseto, em laboratório, em folhas de eucalipto previamente danificadas por lagartas e em folhas sem dano.

\section{Material e Métodos}

Os adultos de T. arnobia, provenientes de coleta no campo em plantios de eucalipto (Eucalyptus grandis) no Município de Três Marias, MG, foram mantidos em condições controladas de laboratório (laboratório de Entomologia Florestal da Universidade Federal de Viçosa) com temperatura de $25 \pm 2^{\circ} \mathrm{C}$, fotófase de 12 horas e umidade relativa de $60 \pm 10 \%$. Os casais foram separados e acondicionados em potes de plástico de $500 \mathrm{~mL}$ com tampa de plástico, furada no centro, vedados por uma tela de malha fina, de organza, para permitir a aeração no recipiente.

Dentro dos potes, foram colocadas tiras de papel presas à tampa para que nelas fossem efetuadas as posturas. Quando as lagartas eclodiram, foram colocadas dentro de caixas de madeira teladas $(0,45 \times 0,45 \times 0,45 \mathrm{~m})$, que passaram a ser utilizadas como gaiolas de criação.

As lagartas foram separadas em dois grupos: um recebia exclusivamente dieta à base de folhas de eucalipto (Eucalyptus cloesiana) e, o outro, folhas de goiaba (Psidium guajava). Esses grupos foram mantidos por quatro gerações dentro das gaiolas. Desta forma, foram obtidas duas populações de T. arnobia, conforme a dieta recebida.

Os insetos eram separados por casais após passarem para a fase adulta, conforme descrito anteriormente, e as lagartas obtidas na quinta geração eram utilizadas nos experimentos realizados no campo e no laboratório no hospedeiro correspondente (goiaba ou eucalipto).

O experimento realizado no campo foi constituído de dois tratamentos (goiaba e eucalipto) e dez repetições, contendo 30 lagartas de $T$. arnobia por repetição.

As lagartas foram colocadas dentro de sacos constituídos de um tecido de tela fino (organza) $(0,70 \times 0,40 \mathrm{~m})$, que foram presos de maneira a envolver galhos com folhas intactas de plantas dos hospedeiros com idade média de 2,5 anos.

O ciclo larval e o número de lagartas mortas foram avaliados por leituras realizadas a cada 72 horas até a fase de pré-pupa. Os resultados obtidos foram analisados por ANOVA.

No teste de resistência induzida em plantas de eucalipto, as lagartas de T. arnobia provenientes de cultura estoque mantida em eucalipto foram separadas no laboratório em dois grupos de 30 indivíduos. Um grupo foi criado em folhas de plantas de Eucalyptus cloesiana previamente atacadas e, o outro, em folhas de plantas de E. cloesiana sem ataque.

Foram consideradas como atacadas previamente, plantas nas quais foram mantidas cinco gerações consecutivas 
de T. arnobia. As plantas utilizadas tinham aproximadamente a mesma idade (2,5 anos). A cada geração, colocava-se 500 lagartas por planta, divididas em dez sacos de organza. As plantas sem ataque prévio eram supervisionadas diariamente durante o período experimental para verificar se não estavam sendo atacadas por outro herbívoro.

As lagartas de cada grupo foram individualizadas em placas de Petri (diâmetro de $15,0 \mathrm{~cm}$ e altura de $1,2 \mathrm{~cm}$ ). Cada placa recebia uma folha da planta correspondente, cujo pecíolo era envolto em algodão umedecido em água destilada com a finalidade de mantê-la túrgida. Diariamente, por ocasião da substituição das folhas, as placas eram limpas, retirando-se as fezes e os resíduos alimentares.

A partir do quinto ínstar, devido ao aumento do volume do corpo, as lagartas eram transferidas para potes de plástico individuais $(500 \mathrm{~mL})$ com tampa com um furo no centro, o qual era vedado por uma tela de malha fina. As lagartas foram mantidas nesses potes até o início do período de pré-pupa. Foram avaliados os números de ínstares, duração do ciclo larval, tamanho das lagartas em cada ínstar, mortalidade, período de pré-pupa, viabilidade pupal e período pupal.

\section{Resultados e Discussão}

Os dados obtidos em campo com T. arnobia, criada sobre dois hospedeiros, mostram que a taxa de mortalidade de lagartas de T. arnobia foi maior em plantas de goiaba do que em plantas de eucalipto. Isto ocorreu em todas as leituras realizadas durante o teste, com as diferenças sendo significativas $(\mathrm{p}<0,05)$ de uma dieta para outra. A porcentagem média total de sobrevivência larval foi de 78,00\% em eucalipto e de 29,33\% em goiaba (Tabela 1). As lagartas de T. arnobia apresentaram ciclo menor quando criadas em plantas de goiaba (27,90 dias) se comparado com lagartas criadas em plantas de eucalipto (30,30 dias) $(\mathrm{p}<0,05)$.

Tabela 1. Sobrevivência e ciclo larval (média \pm desvio-padrão) de Thyrinteina arnobia (Lepidoptera: Geometridae) criada em folhas de Eucalyptus cloesiana (eucalipto) e em folhas de Psidium guajava (goiaba) ${ }^{(1)}$.

\begin{tabular}{lcc}
\hline Parâmetros & Eucalipto & Goiaba \\
\hline Sobrevivência larval (\%) & $78,00 \pm 1,76 \mathrm{a}$ & $29,33 \pm 1,43 \mathrm{~b}$ \\
Ciclo larval (dias) & $30,30 \pm 0,38 \mathrm{a}$ & $27,90 \pm 0,18 \mathrm{~b}$ \\
\hline \multicolumn{3}{l}{ Médias seguidas de mesma letra, na linha, não diferem entre si pelo } \\
teste F, a 5\% de probabilidade.
\end{tabular}

Estes resultados revelaram que a goiaba não é um bom hospedeiro para o desenvolvimento populacional de T. arnobia, possivelmente pelos mecanismos de defesa deste hospedeiro (defesas químicas que agem diretamente sobre o herbívoro), que interferem negativamente na performance desse inseto.

Resultados contrários aos obtidos no presente trabalho foram encontrados por Santos et al. (2000). Segundo esses autores, o hospedeiro de origem (goiaba) seria o melhor hospedeiro para desenvolvimento e manutenção da população de T. arnobia. Talvez as diferenças encontradas nos dois trabalhos sejam devidas ao fato de que, no presente trabalho, as plantas utilizadas foram submetidas à herbivoria de T. arnobia por cinco gerações sucessivas. Deste modo, houve, simultaneamente, indução do sistema de defesa das plantas e adaptação das lagartas, que foram retiradas de plantios de eucalipto e criadas em plantas de goiaba. Entretanto, no trabalho de Santos et al. (2000), esse método não foi executado, pois os insetos foram coletados de eucalipto e os testes, feitos com a primeira geração em goiaba.

Outros aspectos referentes ao cultivo ou ocorrência dos hospedeiros citados, exceto a capacidade fisiológica das plantas de favorecerem a ocorrência de surtos em plantios de eucalipto, são as extensas áreas utilizadas nesses plantios, que proporcionam maior disponibilidade de alimento no estabelecimento de população de T. arnobia. Em monoculturas como essa, há em geral uma redução intensa da fauna e da flora, acarretando menor densidade de inimigos naturais com maior dificuldade de se estabelecerem.

É possível que T. arnobia possa ter migrado de seu hospedeiro de origem para outros hospedeiros como eucalipto a fim de fugir da pressão que inimigos naturais exercem sobre sua população. Segundo Holtz (2001), o percejevo predador Podisus nigrispinus é mais eficiente na procura por herbívoros em plantas de goiaba do que em plantas de eucalipto. Os resultados foram obtidos oferecendo odores do complexo planta-herbívoro com a finalidade de estudar o forrageamento de percevejos predadores.

Porém, outros parâmetros podem estar relacionados com a adaptação de T. arnobia em plantios 
de eucalipto. Como observado, a mortalidade larval no campo foi alta em plantas de goiaba $(70,77 \%)$ (Tabela 1), sendo semelhante aos resultados de Holtz (2001), obtidos em laboratório (75,00\%). Isto demonstra que plantas de goiaba possuem mecanismos de defesa constitutivos que desfavorecem o estabelecimento e desenvolvimento de T. arnobia e, para fugir dessa pressão, provavelmente esse herbívoro migrou para outros hospedeiros como o eucalipto, onde se adaptou por conseguir vencer as barreiras de defesa.

Aparentemente, T. arnobia está suplantando o sistema de defesa químico das plantas de eucalipto. $\mathrm{O}$ inseto conseguiu estabelecer suas populações nesse hospedeiro $(70,77 \%$ de mortalidade larval em plantas de goiaba e $12,00 \%$ de mortalidade em plantas de eucalipto). Segundo Maurício \& Rausher (1997), as defesas químicas das plantas podem atuar como repelentes e também reduzir a digestibilidade de insetos herbívoros, além de afetar a sua performance no hospedeiro e seu desenvolvimento na população atual, bem como nas próximas gerações. Porém, os herbívoros podem evitar plantas com os mecanismos de defesa letal, procurando outros hospedeiros que, possivelmente, não afetam sua performance.

Quanto à biologia de T. arnobia, em laboratório, todos os parâmetros apresentaram diferença significativa $(p<0,05)$ entre a dieta "plantas de eucalipto danificadas previamente por lagartas de T. arnobia" e "plantas de eucalipto sem danos (limpas)", exceto quanto ao parâmetro de período de pré-pupa (Tabela 2).

O comprimento de lagartas de T. arnobia foi maior quando criadas com folhas de plantas de eucalipto sem danos $(3,47 \mathrm{~cm})$ do que com danos
$(3,00 \mathrm{~cm})$ (Tabela 2$)$, porém, a diferença significativa $(\mathrm{p}<0,05)$ só foi observada no sexto ínstar (Figura 1). O ciclo larval de T. arnobia foi menor em eucalipto previamente atacado (32,89 dias) em relação ao eucalipto sem ataque (36,39 dias) (Tabela 2). A mortalidade larval também foi significativamente maior em eucalipto previamente atacado $(30,00 \%) \mathrm{em}$ relação a plantas de eucalipto sem ataque $(10,00 \%)$ (Tabela 2), apresentando maiores porcentagens de mortalidade em todos os ínstares (Figura 2).

A viabilidade pupal de T. arnobia foi maior em plantas de eucalipto sem danos $(90,00 \%)$ do que em plantas de eucalipto previamente danificadas (73,33\%) por lagartas de T. arnobia (Tabela 2). O ciclo pupal também apresentou melhor desenvolvimento na dieta com plantas de eucalipto sem danos (8,04 dias) do que nas plantas com danos (9,38 dias).

Nos testes realizados em campo, observa-se que plantas de eucalipto são susceptíveis ao ataque de T. arnobia quando comparadas com o seu hospedeiro de origem (goiaba). No entanto, realizando-se testes que comparam plantas de eucalipto que foram danificadas por lagartas de T. arnobia durante cinco gerações consecutivas com plantas da mesma espécie sem danos (limpas), observou-se que a herbivoria foliar nas plantas de eucalipto resultou em respostas de defesa das plantas ao ataque prévio do herbívoro. A resposta de defesa obtida afetou o desenvolvimento do herbívoro.

As respostas das plantas após a herbivoria, ou seja, mortalidade larval de $30,00 \%$ em plantas danificadas e $10,00 \%$ em plantas sem danos, e viabilidade pupal de 73,33\% em plantas danificadas e $90,00 \%$ (Tabela 2) em plantas sem danos, demons-

Tabela 2. Aspectos biológicos (média \pm desvio-padrão) de Thyrinteina arnobia (Lepidoptera: Geometridae) criada em folhas de Eucalyptus cloesiana danificadas previamente por lagartas de T. arnobia e em folhas de eucalipto sem danos.

\begin{tabular}{lcc}
\hline Parâmetros & Eucalipto com danos & Eucalipto sem danos \\
\hline Ciclo larval (dias) & $32,89 \pm 0,26 \mathrm{~b}$ & $36,39 \pm 0,47 \mathrm{a}$ \\
Mortalidade larval (\%) & $30,00 \pm 0,61 \mathrm{a}$ & $10,00 \pm 0,44 \mathrm{~b}$ \\
Comprimento da lagarta (cm) & $3,00 \pm 0,35 \mathrm{~b}$ & $3,47 \pm 0,56 \mathrm{a}$ \\
Período de pré-pupa & $2,24 \pm 0,45 \mathrm{a}$ & $2,26 \pm 0,81 \mathrm{a}$ \\
Viabilidade pupal $(\%)$ & $73,33 \pm 1,32 \mathrm{~b}$ & $90,00 \pm 1,32 \mathrm{a}$ \\
Ciclo pupal (dias) & $9,38 \pm 1,07 \mathrm{a}$ & $8,04 \pm 0,76 \mathrm{~b}$ \\
\hline
\end{tabular}

${ }^{(1)}$ Médias seguidas de mesma letra, na linha, não diferem entre si pelo teste $\mathrm{F}$, a $5 \%$ de probabilidade. 
tram que a resistência induzida afeta a população de herbívoros e são os dados que melhor comprovam a indução do sistema de defesa das plantas utilizadas neste trabalho.

As demonstrações de resistência induzida são pouco comuns, e são necessárias para inferir na di-

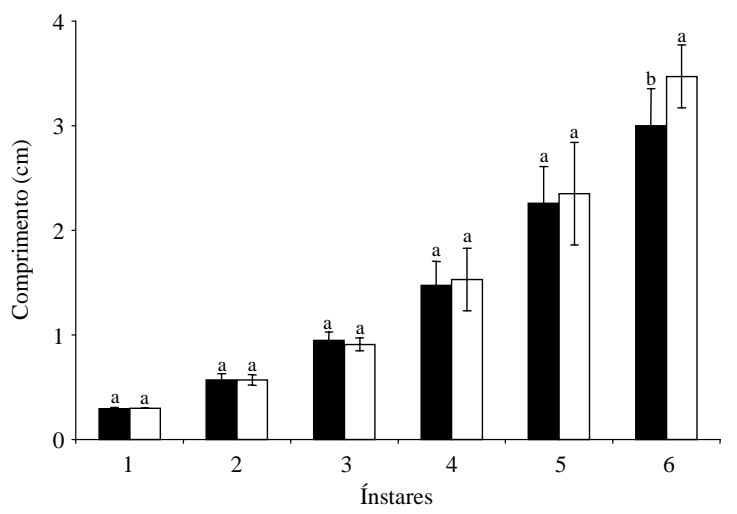

Figura 1. Comprimento de lagartas por ínstar de Thyrinteina arnobia (Lepidoptera: Geometridae) criadas em folhas de plantas de Eucalyptus cloesiana previamente danificadas ( $\mathbf{\square})$ por T. arnobia e em folhas de plantas de E. cloesiana não danificadas ( $\square$ ). Em cada ínstar, médias seguidas com mesma letra não diferem entre si, pelo teste $\mathrm{F}$, a $5 \%$ de probabilidade.

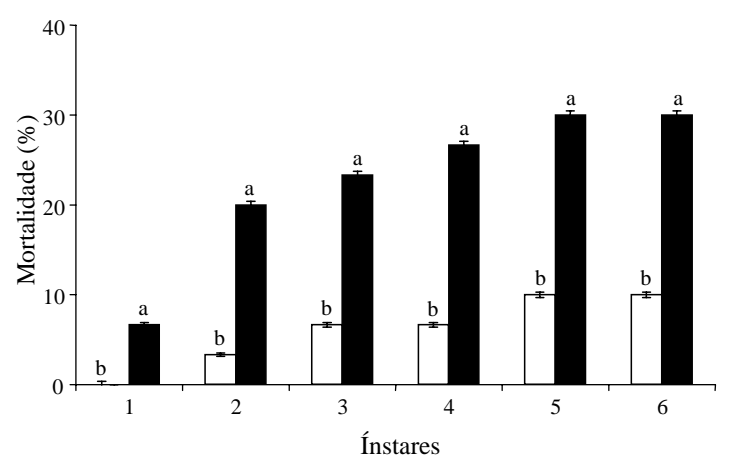

Figura 2. Mortalidade larval de Thyrinteina arnobia (Lepidoptera: Geometridae) criadas em folhas de plantas de Eucalyptus cloesiana previamente danificadas ( $\square$ ) por T. arnobia e em folhas de plantas de E. cloesiana não danificadas ( $\square$ ). Em cada ínstar, médias seguidas com mesma letra não diferem entre si, pelo teste F, a 5\% de probabilidade. nâmica populacional de herbívoros em plantas. Os efeitos da indução na aptidão (fitness) da planta são quase completamente ausentes, porém é certo que afetam a biologia do inseto (Brown, 1988; Karban, 1993; Baldwin, 1991, 1998).

Estudos têm mostrado que a qualidade do alimento afeta a biologia do herbívoro, o que pode estar relacionado com os compostos químicos que as plantas produzem quando danificadas. A resistência induzida é uma mudança na planta que reduz a preferência ou performance de herbívoros que a atacam posteriormente (Baldwin, 1991).

As interações entre herbívoros e plantas são tidas como antagônicas, com cada participante tendo a capacidade para afetar a seleção do outro (McNaughton et al., 1997). Respostas das plantas para herbivoria são vistas como uma estratégia de defesa quase onipresente que, em algumas circunstâncias, provavelmente é adaptável. Porém, as complexidades dos efeitos de herbívoros em plantas e vice-versa ainda estão sendo estudadas. As dificuldades desses estudos aumentam porque as respostas induzidas correspondem ao grau de ataque do herbívoro e são diferentes entre plantas.

\section{Conclusões}

1. Thyrinteina arnobia apresenta dificuldade em se desenvolver e estabelecer em plantas de goiaba.

2. Mesmo quando plantas de eucalipto apresentam indução de defesa, T. arnobia se desenvolve, se adapta e mantém suas populações nessa essência florestal.

3. Esses herbívoros convivem com o sistema de defesa de plantas de eucalipto.

4. No campo, a migração para plantas vizinhas (sem ataque) ou para áreas não atacadas dentro do mesmo plantio pode ser uma das estratégias utilizadas por esses insetos.

5. A estratégia de migrar explica o ciclo dos surtos populacionais de T. arnobia em eucalipto.

\section{Agradecimentos}

Ao Conselho Nacional de Desenvolvimento Científico e Tecnológico e à Coordenação de Aperfeiçoamento de Pessoal de Nível Superior, pela con- 
cessão de bolsas; à Universidade Federal de Viçosa, pelo apoio na realização deste trabalho.

\section{Referências}

AGRAWAL, A. A. Specificity of induced resistance in wild radish: causes e consequences for two specialist and two generalist caterpillars. Oikos, Cambridge, v. 89, p. $493-$ 500,2000

ANJOS, N.; SANTOS, G. P.; ZANUNCIO, J. C. Pragas do eucalipto e seu controle. Informe Agropecuário, Belo Horizonte, v. 12, p. 50-58, 1986

BALDWIN, I. T. Damage induced alkaloids in wild tobacco. In: RAUPP, M. J.; TALLAMY, D. W. (Ed.). Phytochemical induction by herbivores. New York: Wiley, 1991. p. 427-438.

BALDWIN, I. T. Jasmonate induced responses are costly but benefit plants under attack in native populations. Proceedings of the National Academy of Sciences of the United States of America, Washington, v. 95, p. 81138118, 1998.

BROWN, D. G. The cost of plant defense: an experimental analysis with inducible proteinase inhibitors in tomato. Oecologia, Berlin, v. 76, p. 467-470, 1988.

DICKE, M. Volatile spider mite pheromone and host plant kairomone, involved in spaced out gregariousness in the spider mite Tetranychus urticae. Physiological Entomology, Oxford, v. 11, p. 251-262, 1986.

FOX, L. R.; MacAULEY, B. J. Insect grazing on Eucalyptus in response to variation in leaf tannins and nitrogen. Oecologia, Berlin, v. 29, p. 145-162, 1977.

HAGEN, K. S.; DADD, R. H.; REESE, J. The food of insects In: HUFFAKER, C. B.; RABB, R. L. (Ed.). Ecological Entomology. New York: J. Wiley, 1986. p. 79-112.
HOLTZ, A. M. Interações tritróficas afetando os surtos de pragas em Myrtaceae. 2001. 104 f. Dissertação (Mestrado em Entomologia) - Universidade Federal de Viçosa, Viçosa, MG, 2001.

KARBAN, R. Costs and benefits of induced resistance and plant density for a native shrub, Gossypium thurberi. Ecology, New York, v. 74, p. 9-19, 1993.

MAURICIO, R.; RAUSHER, M. D. Variation in the defense strategies of plants: are resistance and tolerance mutually exclusive? Ecology, New York, v. 78, p. 13011311, 1997.

McNAUGHTON, S. J.; BANYIKWA, F. F.; MCNAUGHTON, M. M. Promotion of the cycling of diet enhancing nutrients by African grazers. Science, Washington, v. 278, p. 1798-1800, 1997.

PRICE, P. W. Insect ecology. $3^{\text {rd }}$ ed. New York: J. Wiley, 1997. $874 \mathrm{p}$.

PRICE, P. W. Semiochemicals in evolutionary time. In: NORLUND, D. A.; JONES, R. L.; LEWIS, W. J. (Ed.). Semiochemicals: their role in pest control. New York: Wiley, 1981. p. 251-279.

SANTOS, G. P.; ZANUNCIO, T. V.; ZANUNCIO, J. C. Desenvolvimento de Thyrinteina arnobia Stoll (Lepidoptera: Geometridae) em folhas de Eucalyptus urophylla e Psidium guajava. Anais da Sociedade Entomológica do Brasil, Rio de Janeiro, v. 29, p. 13-22, 2000.

VET, L. E. M.; DICKE, M. Ecology of infochemical use by natural enemies in a tritrophic context. Annual Review Entomology, Palo Alto, v. 37, p. 141-172, 1992.

ZANUNCIO, J. C.; FAGUNDES, M.; ANJOS, N.; ZANUNCIO, T. V.; CAPITANI, L. C. Levantamento e flutuação populacional de lepidópteros associados à eucaliptocultura - V: Região de Belo Oriente, MG, junho de 1986 a maio de 1987. Revista Árvore, Viçosa, MG, v. 1 , p. $35-44,1990$. 